\title{
Schmerz im biopsychosozialen Modell
}

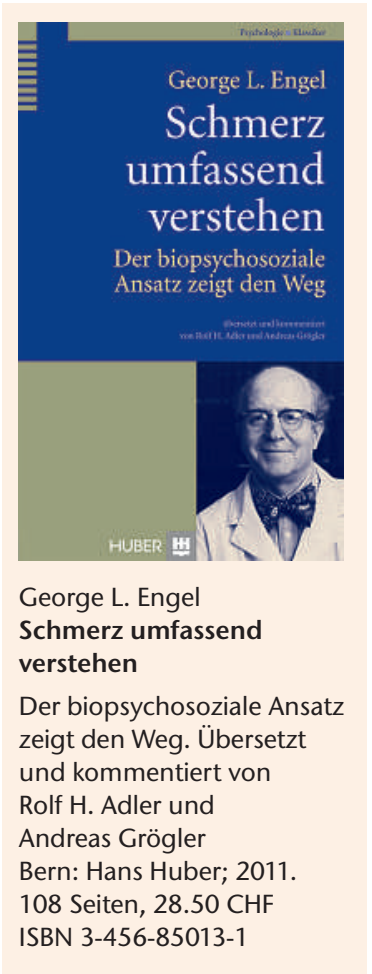

Prof. Rolf Adler, Internist und Psychoanalytiker, setzt sich seit seinen Anfängen im Arztberuf für die Umsetzung des biopsychosozialen Konzeptes in die praktische Medizin ein. Biopsychosozial meint Erfassung, Gewichtung und Integration von körperlichen, psychischen und sozialen Faktoren in Diagnose und Therapie. Das Anliegen ist einfach und klar, droht aber im Zuge der gewaltigen medizinischtechnischen Fortschritte in den letzten fünf Jahrzehnten mehr und mehr unterzugehen.

Rolf Adler gibt nicht auf. Nachdem er als Chefarzt und Professor für Innere Medizin am Inselspital in Bern während über zwanzig Jahren den biopsychosozialen Ansatz in Praxis, Lehre und Forschung umgesetzt hat, besinnt er sich jetzt, zehn Jahre nach seiner Emeritierung, auf seine Wurzeln. Zusammen mit Andreas Grögler präsentiert und kommentiert er zwei grundlegende und wegweisende Arbeiten seines Lehrers George Engel in deutscher Übersetzung: Psychogenic pain and the pain prone patient, $1959 \mathrm{im}$ American Journal of Medicine publiziert, und The need of a new medical model, a challenge for biomedicine, 1977 in Science erschienen. Wissenschaftliche Erkenntnisse in der Medizin sind in der Regel kurzlebig. Was mehr als zehn Jahre zurückliegt, gilt oft als überholt. Umso erstaunlicher, dass die vor über fünfzig, resp. über dreissig Jahren publizierten Arbeiten bis heute nichts an Aktualität eingebüsst haben.
Die Abhandlung über Entstehung und Persistenz von psychogenen Schmerzen aufgrund von traumatischen Erfahrungen ist reich mit eindrücklichen klinischen Beispielen ausgestattet und belegt Engels subtile Beobachtungsgabe, seine der Zeit entsprechend grossen Kenntnisse in Neurophysiologie und vor allem der psychosozialen Entwicklung des Menschen. In seinem scharfsinnigen Kommentar belegt Adler, dass die 1959 aufgestellten Hypothesen in zahlreichen kontrollierten Studien zu einem grossen Teil bestätigt werden konnten.

Die zweite Arbeit über die Notwendigkeit eines biopsychosozialen Modells in der Medizin ist theoretischer Natur. Sie liefert gewissermassen die wissenschaftstheoretische Grundlage zu den Beobachtungen und Hypothesen in der Arbeit über psychogenen Schmerz und die Neigung, Schmerz leiden zu müssen. Schritt für Schritt und sehr anschaulich baut Engel eine solide Brücke über den Graben des Körper-Seele-Dualismus, der das Mentale vom Körperlichen scharf trennt. Es wird offensichtlich, dass disease (Krankheit als objektive Veränderung im Körper) und illness (Krankheit als subjektives Erleben) zwingend zusammengehören und sich gegenseitig beeinflussen und somit der biomedizinische Ansatz zum biopsychosozialen Verständnis von Gesundheit und Krankheit ausgeweitet werden muss.

In seinem witzigen Kommentar erläutert Adler das 
biopsychosoziale Modell anhand einer kleinen Kriminalgeschichte und ergänzt die theoretischen Überlegungen von Engel mit dem Einbezug von Konstruktivismus und Semiotik.

Engels Stil ist zeitweise etwas kompliziert, und manche Stellen musste ich zweimal lesen, um sie zu verstehen, was nicht unbedingt schadet, weil man so der Sache auf den Grund gehen muss. Stellenweise ist die Über- setzung etwas unbeholfen, stört aber den Lesefluss nicht. Jedenfalls habe ich das Büchlein mit Interesse und Vergnügen gelesen und mich über die Aktualität der Beiträge gefreut. Das Freudzitat von Engel: «Erlaube dem Patienten frei zu sprechen und nimm ernst, was der Patient sagt», gilt heute mehr denn je.

Prof. em. Dr. med. Christoph Hürny, St. Gallen 